\title{
The relevance of EGFR overexpression for the prediction of the malignant transformation of oral leukoplakia
}

\author{
JUTTA RIES $^{1}$, ELEFTHERIOS VAIRAKTARIS ${ }^{3}$, ABBAS AGAIMY $^{2}$, MORITZ BECHTOLD $^{1}$, \\ PATRICIA GORECKI ${ }^{1}$, FRIEDRICH W. NEUKAM ${ }^{1}$ and EMEKA NKENKE ${ }^{1}$ \\ ${ }^{1}$ Department of Oral and Maxillofacial Surgery, and ${ }^{2}$ Institute of Pathology, Erlangen University Hospital, \\ D-91054 Erlangen, Germany; ${ }^{3}$ Department of Oral and Maxillofacial Surgery, University of Athens, Athens, Greece
}

Received March 26, 2013; Accepted May 2, 2013

DOI: $10.3892 /$ or.2013.2545

\begin{abstract}
The present study evaluated the relevance of EGFR overexpression in prediction of malignant transformation of oral leukoplakia (OLP). The retrospective study comprised paraffin-embedded tissue samples of OLP that transformed into oral squamous cell carcinoma (OSCC) $(n=53)$ and tissue samples of OLP that did not transform into OSCC $(n=45)$ during a follow-up period of 5 years. EGFR overexpression was assessed immunohistochemically. A significantly different expression rate of EGFR was determined between transformed and non-transformed OLP $(\mathrm{P}=0.017)$. A statistically significant increase of EGFR expression for low dysplasia lesions in group I compared to group II was proven (D0, $\mathrm{P}=0.013$; $\mathrm{D} 1, \mathrm{P}=0.049$ ). By calculation of ROC curve and determination of highest Youden index the optimal threshold value [cut-off point $(\mathrm{COP})=44.96]$ for distinguishing the transformed from non-transformed lesions was estimated (critical expression rate of EGFR). Using the determined COP the correlation between high-risk lesions and the detection of increased expression rates were significant $(\mathrm{P}=0.001)$. In the future, the assessment of EGFR overexpression in OLP may allow identifying OLP lesions with an increased risk of malignant transformation that may have been regarded harmless when only the grade of dysplasia had been taken into account.
\end{abstract}

\section{Introduction}

Oral squamous cell carcinoma (OSCC), one of the most common oral malignancies, presents a clinical diagnostic challenge, particularly in its early stages of development. Nevertheless, the early diagnosis of oral cancer plays a key role

Correspondence to: Dr Jutta Ries, Department of Oral and Maxillofacial Surgery, Erlangen University Hospital, Glueckstrasse 11, D-91054 Erlangen, Germany

E-mail: jutta.ries@uk-erlangen.de

Key words: oral squamous cell carcinoma, EGFR overexpression, immunohistochemistry, oral leukoplakia, malignant transformation in patient survival. While early stages of OSCC are curable in $80 \%$ of the cases, the 5 -year survival rate drops to $30 \%$ in advanced stages and corresponds, finally, to a rate of $50 \%$. Unfortunately, still two thirds of the patients suffering from OSCC are diagnosed in an advanced stage resulting in a 5-year survival rate of $\sim 50 \%$. Hence, methods for early diagnosis of the disease are urgently needed (1-3).

A second problem in development of oral carcinomas is that $\sim 67 \%$ of them are preceded by oral potentially malignant disorders mostly by leukoplakia (OLP) which represents the most common potentially malignant lesion of the oral mucosa. Statistically, $1-18 \%$ of these lesions show malignant transformation to OSCC (4-7). However, like early diagnosis of the malignancy the evaluation of cancer risk coming up from individual lesions is difficult. To date, histopathological assessment of the degree of dysplasia is the basis for the prediction of malignant transformation $(4,5,8,9)$. Unfortunately, this method is subjective and lacks intra- and inter-observer reproducibility (10). In addition, it was found that histological features alone cannot accurately predict whether these tissue changes of the oral mucosa remain stable, regress or progress to malignancy and sometimes even non-dysplastic lesions suddenly show malignant transformation (10).

Therefore, attempts have been made to develop diagnostic and prognostic biomarkers and methods for monitoring premalignant stages (7). Specific molecular biological chances in oral tissues hold great promise in this respect and in the past attempts were made to develop methods based on specific molecular markers for more accurate assessment of cancer risk of OPL. However, so far none of these markers has been introduced to clinical routine (7,11-22).

Aberrant expression and function of molecules involved in signalling networks controlling cell cycle, differentiation, apoptosis, genomic stability, motility, angiogenesis and metastasis have been considered as biomarkers for risk assessment of malignant transformation $(5,9)$.

The epithelial growth factor (EGFR) regulates the signalling involved in cell proliferation and differentiation. It is overexpressed in several tumors including OSCC. There is substantial, but not clear evidence that high expression of EGFR is correlated with advanced tumor stages, metastases and poor clinical outcome $(23,24)$. 
Enhanced expression of the EGFR family has been detected in proliferative cells of the basal layer in normal oral mucosa and leukoplakia with mild, moderate and severe dysplastic lesions. Some studies report that the pattern of EGFR expression in dysplastic leukoplakia coincides with the histological assessment of dysplasia in these lesions and they have indicated that EGFR upregulation may be a useful marker for identifying individuals at risk of OSCC development $(7,18,24-28)$.

To date, there is a lack of information on the rate of malignant transformation of OLP over longer follow-up intervals comparing immunohistochemical features and conventional histopathological aspects of the lesions. Therefore, the present study included only OLP lesions of patients with 5-year follow-up data. The aim of the present study was to evaluate if there were correlations between EGFR overexpression in OLP, the rate of malignant transformation and the histopathological grade of dysplasia.

\section{Materials and methods}

Tissue samples and patients. The study was approved by the Ethics Committee of the University of Erlangen-Nuremberg and patients' informed consent was obtained. A total of 148 tissue samples (paraffin blocks) were included in the study that had been collected at the Department of Pathology, University Hospital Erlangen between 1997 and 2011. Group I consisted of 53 OLP samples that showed malignant transformation into OSCC during the 5-year follow-up. Group II included 45 OLP samples that did not show a malignant transformation in the 5-year follow-up. Group III included 30 samples of sound oral mucosa (NOM) of healthy volunteers and served as negative control. Group IV consisted of 21 OSCC samples that were the result of malignant transformation of OLP of group I. Basic patient data including age and gender were collected. Moreover, the time interval between diagnosis of OLP and the diagnosis of OSCC (disease-free survival time, DFS) was determined.

Oral epithelial dysplasia classification and tumor histopathology and staging. All samples were evaluated by two pathologists to ensure consistent results. The grade of dysplasia of the OLP samples was histopathologically classified according to WHO classification 2005. Based on architectural, certain histological and cytological features dysplasia of tissues is divided into the stages D0 for no, D1 for mild, D2 for moderate and D3 for severe dysplasia (8). Ideally, diagnosis corresponds to the nature of the lesion meaning that the level of epithelial dysplasia implies the risk of progression into malignancy (D0, no risk; D1, low risk; D2, moderate risk; and D3, severe risk) (9). Clinical staging and TNM classification were done for each tumor patient developing a malignancy based on the primary precursor lesion according to UICC. The OSCC were also classified according to WHO for loss of differentiation as G1, G2 and G3 for well, moderately and poor differentiation, respectively. All biopsies were evaluated by two pathologists to ensure consistent results. Clinical staging (stages I-IV) and classification according to early (including stages I and II) and late (including stages III and IV) clinical stages were recorded.
Immunhistochemical staining of EGFR-1 expression. Immunohistochemical staining was performed with the alkaline phosphatase-anti-alkaline phosphatase method and an automated staining device (Autostainer plus; DakoCytomation, Hamburg, Germany). On all samples the Dako labeled streptavidin biotin alkaline phosphatase system kit (Dako K5005; Dako Diagnostic GMBH, Hamburg, Germany) was applied. EGFR expression was detected by the murine monoclonal antibody M3563 (dilution 1:300; Dako Diagnostic) directed against EGFR-1. Serial sections of $4 \mu \mathrm{m}$ thickness were taken on silanized slides for IHC. Paraffin tissue sections were first dewaxed with xylene (30 min) and rehydrated gradually with ethanol and water. Antigen retrieval applying Proteinase K for 5 min (Dako S3020; Dako Diagnostic) and the whole staining was done using the DakoCytomation Autostainers plus (Dako Diagnostic) according to the recommends of the distributor. After staining the slides were gently rinsed with distilled water and counterstained with haemalaun (Dako S3301; Dako Diagnostic) manually, and then washed gently under running water for 5 min and mounted in aquatex (Merck, Darmstadt, Germany) using cover slips.

Presence of red coloured end product at the site of target antigen was indicative of positive reactivity. Membranous and/or cytoplasmatic staining was estimated as positive result. An unrelated murine IgG1 antibody at the same concentration as the test antibody was used as a negative control.

Semi-quantitative immunohistochemical analysis. We performed semi-quantitative analysis of the membranous and cytoplasmatic expression of EGFR to determine the labelling index of the tissues which is defined as the percentage of expressing cells (ratio of positively stained cells to the total number of cells per ROI, multiplied by 100). Therefore, sections of OLP, OSCC and healthy tissues were first qualitatively evaluated under a bright light microscope (Axioskop; Carl Zeiss, Jena, Germany) at magnifications of $\mathrm{x} 20$ and $\mathrm{x} 40$. For each sample two or three regions of interest (ROI) which span (if possible) the whole epithelium and include 200-300 cells were selected, digitized and documented with a CCD camera (Axiocam 5; Carl Zeiss) and Axiovision software (magnification x 200-x400; Axiovison, Carl Zeiss). Cell counting was performed by two independent observers who were blinded to the tissue origin. Afterwards, the ratio of positively stained cells to the total number of cells per ROI, i.e. the labelling index, was defined.

Statistics. The labelling index per ROI of positively stained cells was used to analyze statistically relevant differential expression rates of EGFR determined by immunohistochemical staining. For the data results three analyzed ROIs were pooled. The labeling indices data were expressed as the median (ME), the interquartile range (IQR), standard deviation (SD) and range. Graphical diagrams are plotted as Box-Whisker Plots which represent the median, the interquartile range, the minimum and the maximum values of determined EGFR expression.

To investigate from which value increased expression is relevant for the likelihood of transformation a cut-of point (COP) was determined. For this a ROC (receiver operating characteristic) curve was established and highest Youdan 
Table I. Distribution of dysplasia in total samples and the two groups and disease-free survival time (DFS) of the patients.

\begin{tabular}{|c|c|c|c|c|c|}
\hline & \multicolumn{5}{|c|}{ Grade of dysplasia } \\
\hline & $\begin{array}{c}\text { D0 } \\
\mathrm{N}(\%)\end{array}$ & $\begin{array}{c}\text { D1 } \\
\mathrm{N}(\%)\end{array}$ & $\begin{array}{c}\text { D2 } \\
\mathrm{N}(\%)\end{array}$ & $\begin{array}{c}\text { D3 } \\
\mathrm{N}(\%)\end{array}$ & $\begin{array}{c}\text { Total } \\
\mathrm{N}\end{array}$ \\
\hline Group I & $15(28.3)$ & $14(26.4)$ & $13(24.5)$ & $11(20.8)$ & 53 \\
\hline Group II & $22(48.9)$ & $19(42.2)$ & $4(8.9)$ & $0(0)$ & 45 \\
\hline \multirow[t]{2}{*}{ Total } & $37(37.8)$ & $32(33.7)$ & $17(17.3)$ & $11(11.2)$ & 98 \\
\hline & \multicolumn{4}{|c|}{ Mean value (month) } & \\
\hline \multirow[t]{3}{*}{ DFS in group I } & 17.4 & 29.6 & 11.7 & 8.5 & 16.8 \\
\hline & \multicolumn{2}{|c|}{ Mean DSF } & \multicolumn{2}{|c|}{ Mean DSF } & $\mathrm{P}<0.05^{\mathrm{a}}$ \\
\hline & \multicolumn{2}{|c|}{23.5} & \multicolumn{2}{|c|}{10.5} & \\
\hline
\end{tabular}

The risk of malignant transformation increases with grade of dysplasia. Most of D2 and all D3 LP proceeded into a tumor. Tumors also developed in OLP with no, or mild dysplasia. Mean value of DFS was lower for patients suffering from high grade dysplasia. ${ }^{\mathrm{a}} \mathrm{Man}-\mathrm{Whitney} \mathrm{U}$ test.
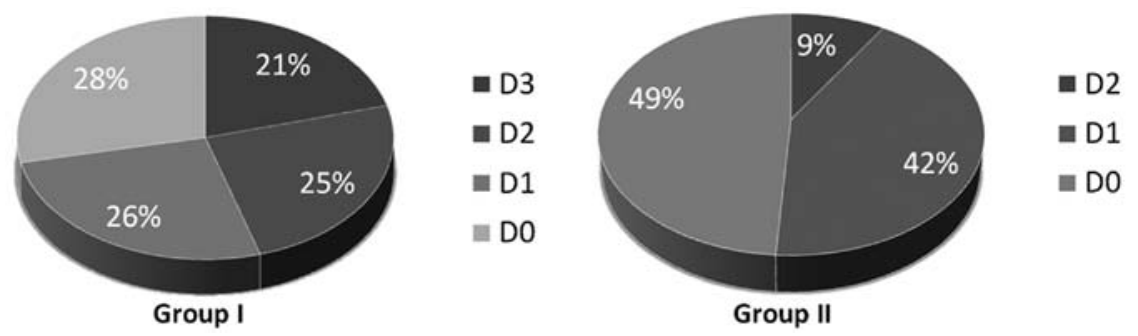

Figure 1. Distribution of cases of OLP into different grades of dysplasia. (A) Number of the different grades of dysplasia in group I (n=53). All grades of dysplasia are represented and there is no prevalence for any dysplasia level within this group. (B) Distribution of dysplasia in non-proceeding leukoplakia (group II). Lesions with high grade of dysplasia are under-represented. There is no prevalence for D0 or D1 lesions within this group.

index and its associated COP (optimal threshold value) were calculated. If the value of a patient lies above the COP, the likelihood of malignant transformation is considered as high. Based on this COP group I and II was divided into two subgroups which show an expression rate over or under the COP. Then the odds ratio and the $95 \%$ confidential interval was calculated.

Comparisons were performed between groups I and II, OSCC and groups I and II and healthy mucosa (group IV) and the groups I, II and II, respectively. Association between malignant transformation and overexpression of EGFR was analysed by Man-Whitney $U$ test and by Fischer's exact test. P-values $<0.05$ were considered as statistically significant. All calculations were performed with SPSS 19.0 for Windows (SPSS Inc., Chicago, IL, USA).

\section{Results}

Clinical features, histopathological data and follow-up of the patients. Of the OLP samples $60.2 \%$ (59/98) belonged to male and $39.8 \%$ (39/98) to female patients. The average age of examined patients with OLP was 55.8 years. Thirty-seven of the OLP had no dysplasia (37.8\%), 33 (33.7\%) showed mild, $17(17.3 \%)$ moderate and $11(11.2 \%)$ severe dysplasia (Table I). Transforming lesions are included in each group of dysplasia. Within the proceeding lesions the distribution of the dysplasia was nearly equal, whereas in group II (nonproceeding OLP) predominantly tissues with low degrees and none with severe dysplasia could be seen. Regarding D2 the fraction of the lesion which transformed was three times higher as within the comparison group II (Table I, Fig. 1). With rising grades of dysplasia the risk of malignant transformation increases although the number of emerging malignancies in D0 and D1 was almost equal. Out of 37 and 33 OLP exhibiting no or mild dysplasia $40.5 \% \quad(n=15)$ and $42.4 \%(n=19)$ proceeded into carcinoma, respectively. In contrast, $76.5 \%(13 / 17)$ of all lesions showing moderate dysplasia and all 11 (100\%) OLP graded as D3 were base of a malignancy (Fig. 2). Statistical investigation revealed that despite of the preselecting of transforming lesions the correlation between risk of tumor development and grade of dysplasia was significant $(\mathrm{P}<0.01)$.

The OLP lesions were divided into two groups. Group I includes 53 lesions proceeding into malignancy within 5 years and 45 samples of group II did not show any malignant transformation (Table I).

Out of the 53 proceeding OLP 40 were found in the oral cavity and 13 in the oropharynx; of these, 64.2\% (34/53) were male and $35.8 \%$ (19/53) female. Consequently, the ratio of men to women was about $2: 1$. The average age of the patients was 61.8 years. The average age of the male patients was 60.2 and that of the women 63.3 years. Within group I all grades of 


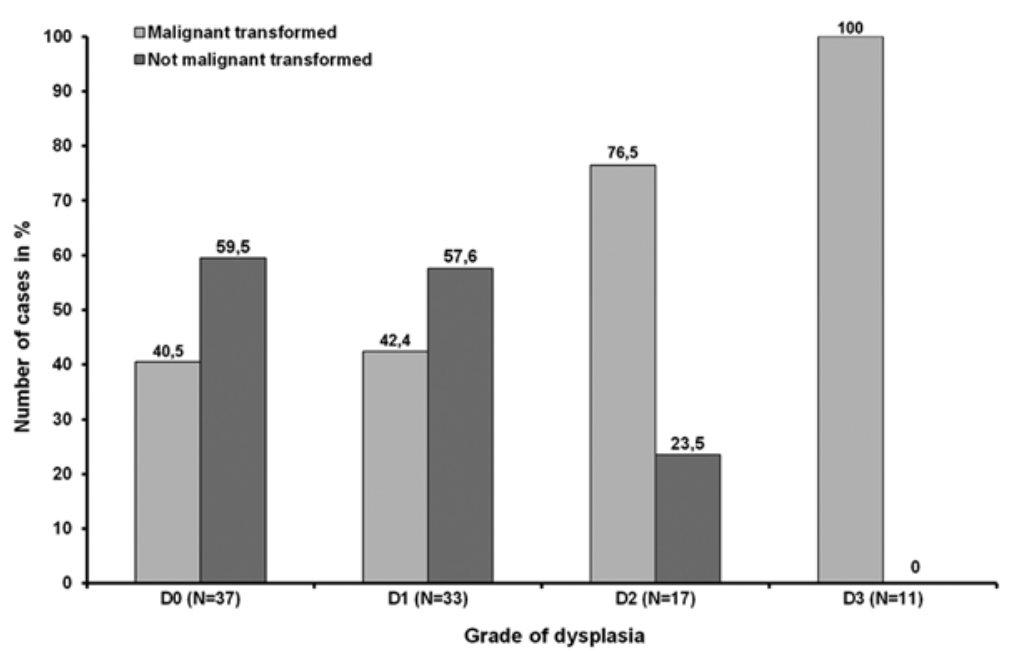

Figure 2. Malignant transformation in relation to grade of dysplasia in total patient contingent ( $\mathrm{n}=98)$. The number of lesions proceeding into carcinoma increases with dysplastic changes. The event of progression was almost identical for D0 and D1 cases. The correlation was significant between transformation and dysplasia. ( $\mathrm{N}$, number of included cases).

Table II. Summary of all calculated P-values determined between the groups.

\begin{tabular}{ll}
\hline Comparison of groups & P-value \\
\hline Group I vs. group II & $\mathbf{0 . 0 1 7}$ \\
Group I vs. group III & 0.08 \\
Group I vs. OSCC & 0.05 \\
Group III vs. group II & 0.3 \\
Group III vs. OSCC & 0.22 \\
\hline
\end{tabular}

Significand differences in expression rates were found between transformed and non-transformed OLP and proceeding OLP and normal healthy mucosa.

dysplasia were represented. Fifteen patients $(28.3 \%)$ had no (D0), 14 (26.4\%) mild, 13 (24.5\%) moderate and 11 (20.8\%) severe dysplasia. There was no preference for any grade of dysplasia within group I (Table I, Fig. 1). The time interval between diagnosis of OLP and detection of malignancy (DFS) was between 10 days and 60 months. The median time from occurrence of OLP until manifestation of OSCC was 16.8 months. The mean values of disease-free survival were lower with higher dysplastic grade. Lesion exhibiting grades of dysplasia higher than D1 transformed to OSCC in a time interval of 10.5 months. Mean DFS value of the group that included D0 and D1 grades of dysplasia was 23.5 months. Because of the small case numbers a statistical analysis was not carried out (Table I).

Group II includes 45 samples which did not transform within the 5-year follow-up period. All samples originated from the oral cavity. Of this group 55.6\% (25/45) were male and $44.4 \%$ (20/45) female. The average age of the patients with OLP was 49.8 years. The average age of the men was 48.3 and that of the women was 51.4 years. Contrary to group I only samples with the degrees of D0 to D2 were included. No OLP with the grade D3 existed; 48.9\% (22/45) showed no dysplasia
Table III. Differences of expression rates within dysplasia groups.

\begin{tabular}{lcccc}
\hline & \multicolumn{4}{c}{ Labelling index/expression rate } \\
\cline { 2 - 5 } Grade of dysplasia & D0 & D1 & D2 & D3 \\
\hline Group I & 45.68 & 44.95 & 28.09 & 39.65 \\
Group II & 27.06 & 26.12 & 37.95 & - \\
\hline
\end{tabular}

Median values of EFGR labelling index within the two groups of OLP according the grade of dysplasia. The highest expression rates of EGFR were observed in D0 and D1 dysplasia of group I. For D2 dysplasia the expression rate was higher in group II. No nontransformed OLP exhibiting D3 dysplasia was included in the study. Expression rate/labelling index, the percentage of expressing cells [ratio of positively stained cells to the total number of cells per ROI (region of interest) multiplied by 100]

(D0), 42.2\% (19/45) mild and 8.9\% (4/45) moderate dysplasia (Table I, Fig. 1). There was no preference for D0 or D1 grades of dysplasia within group II ( $\mathrm{P}>0.05)$. However, samples with high grade of dysplasia were clearly under-represented. The distribution is given in Fig. 2.

Clinical and histological characterization of corresponding OSCC and healthy oral mucosa. Out of the available corresponding tumors 21 could be investigated immunhistologically. Most of the corresponding tumors were small at time of diagnosis. In 15 cases data for tumor size was available; $60 \%$ of all OSCC were $\mathrm{T} 1,13.3 \%$ were $\mathrm{T} 2,6.7 \%$ were $\mathrm{T} 3$ and $6.7 \%$ were $\mathrm{T} 4$ tumors. In 2 cases a carcinoma in situ (CIS) was diagnosed (13.3\%). Twenty OSCC were also graded for differentiation. Five (25\%) OSCC cases were well (G1) and 9 (45\%) were moderately (G2) and $6(30 \%)$ were poorly differentiated. Out of 16 valid samples $81.3 \%$ of the patients who developed a malignancy were diagnosed at early stages of the disease. Only $18.8 \%$ belonged to late stages. 
A Progressing LP
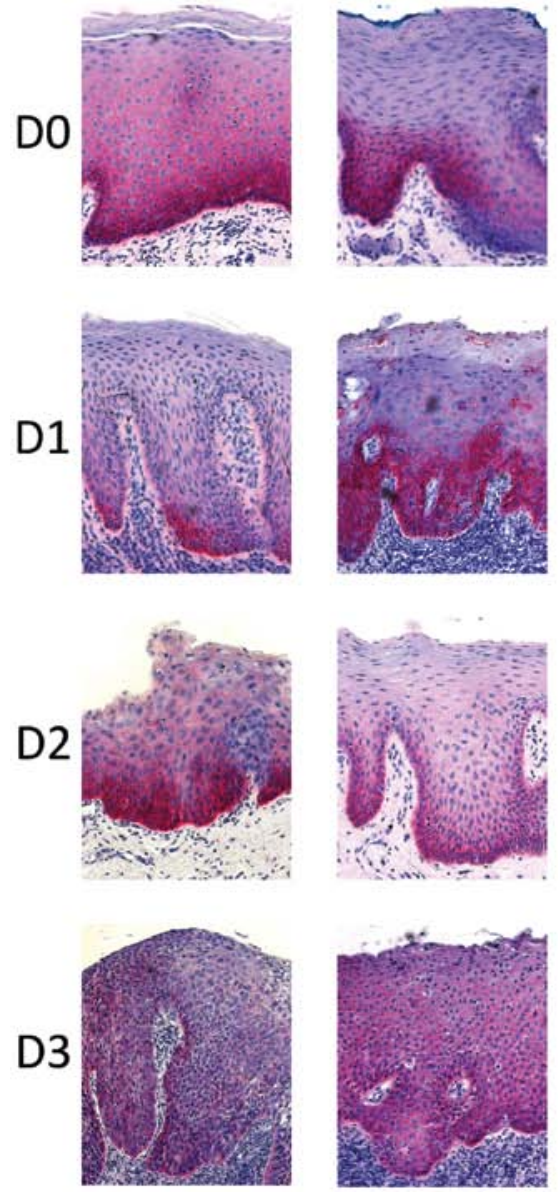

Tumor
B Non progressing LP

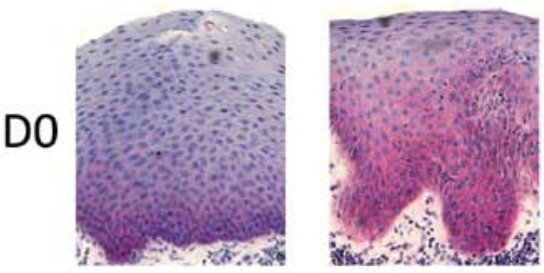

D1
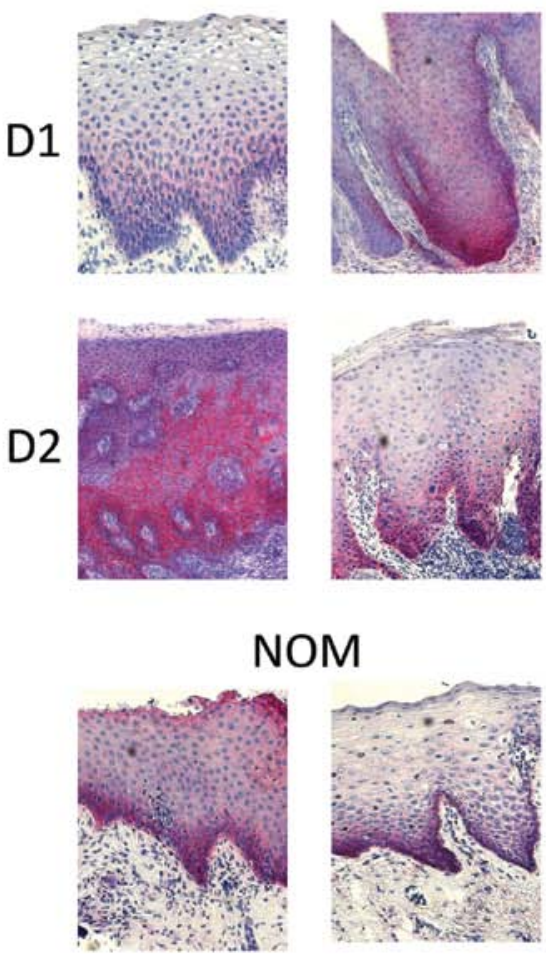
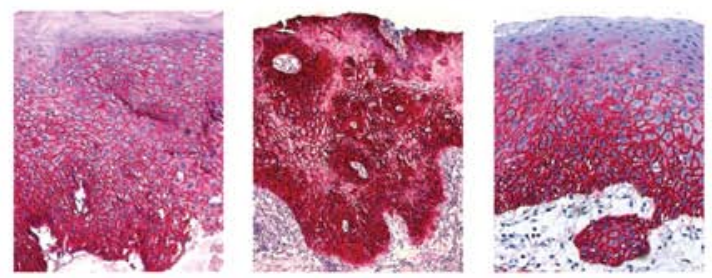

Figure 3. (A) Representative immunohistological staining of progressing leukoplakia (group I) and three corresponding tumors based on such a lesion using an anti-EGFR antibody. The panels show the staining pattern of tissues exhibiting different grades of dysplasia (D0-D3) or malignancy (tumor). (Magnification, x200. (B) Representative immunohistological staining of non-progressing leukoplakia (group II) and normal mucosal samples of a healthy volunteer (NOM). The panels show the staining pattern of tissues exhibiting different grades of dysplasia (D0-D2); normal mucosa (NOM). (Magnification, x200).

The 30 specimens of normal oral mucosa of healthy volunteers did not show remarkable changes, such as inflammation and hyper- or dysplasia, or any clinical abnormalities.

EGFR overexpression in proceeding and non-proceeding leukoplakia and correlation to cancer risk. Representative staining of tissues is shown in Fig. 3. Membranous and cryptoclastic staining could be observed in all types of tissue specimens with different labelling indices. The negative controls demonstrated the absence of non-specific staining (data not shown).

A significant different expression rate could be determined between transformed and non-transformed OLP $(\mathrm{P}=0.017$; Fig. 4). Therefore, overexpression of EGFR is a significant parameter for estimation of cancer risk in OLP. No significant differences in labelling indices could be ascertained for comparison of malignancies with either normal healthy tissues $(\mathrm{P}=0.22)$ or with the OLP which they were based on $(\mathrm{P}=0.50$; Fig. 4). Additionally, no significant difference in expression was found between the control group (group IV) and group I $(\mathrm{P}=0.08)$ and group II $(\mathrm{P}=0.3)$, respectively. All $\mathrm{P}$-values of the comparisons between the different groups are summarized in Table II.

In addition, the mean expression level of EGFR in OLP showing no histological (D0) or mild (D1) changes proceed into cancer was highly different from non-transformed lesions. This was not observed for the small group of D2 dysplasia $(n=4$; Table III). Accordingly, a statistically significant 


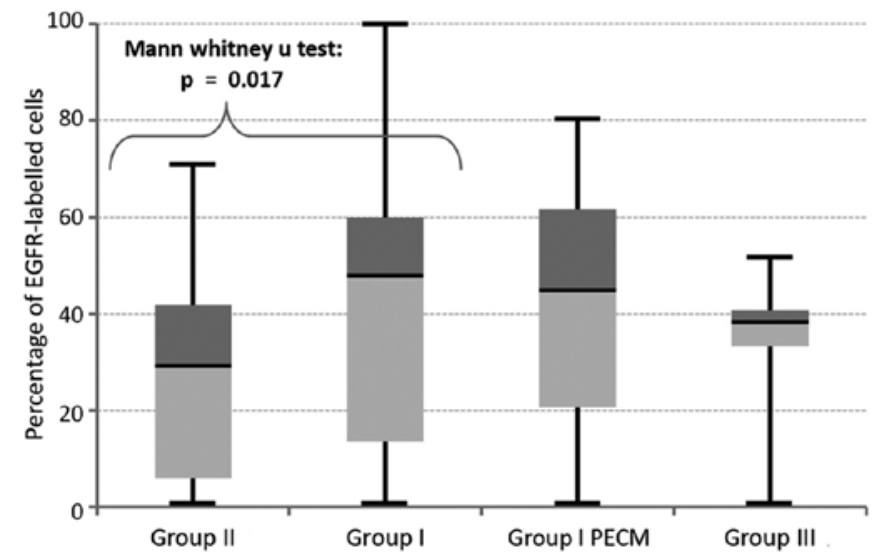

Figure 4. Box-Whisker Plots of median expression rates of EGFR in the four groups. The median, the interquartile range and the standard deviation are given. The expression was increased in transformed compared to non-transformed OLP $(\mathrm{P}=0.017)$. The expression rate was not statistically different between normal healthy tissues (NOM) and transformed $(\mathrm{P}=0.08)$ and nonproceeding OLP $(\mathrm{P}=0.3)$, respectively. The expression rate does not differ in OSCC compared either to NOM or to proceeding OLP $(\mathrm{P}=0.22 ; \mathrm{P}=0.5)$.

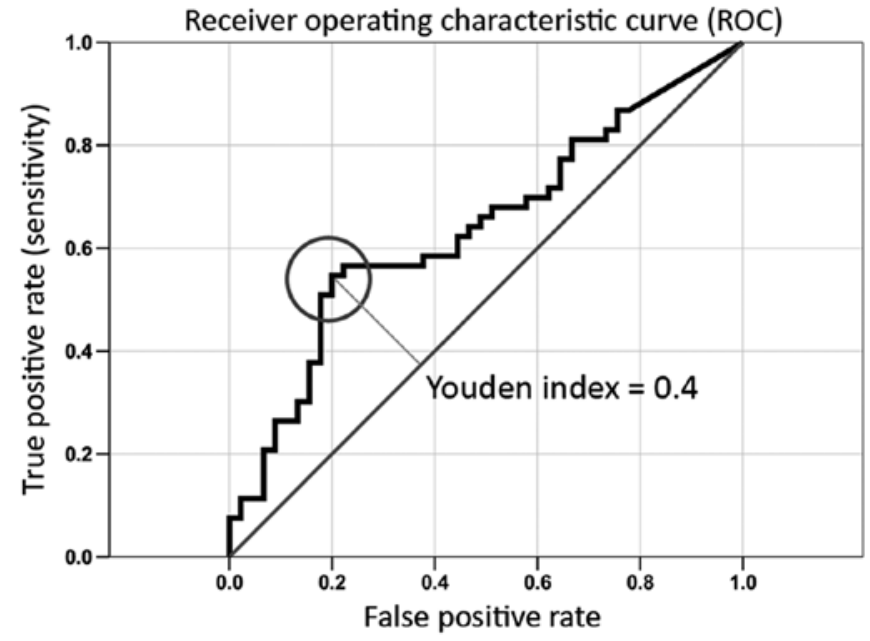

Figure 6. ROC (receiver operating characteristic) curve. The diagram is a plot of the sensitivity (true positive rate) vs. 1-specifity (false positive rate) over all possible threshold values of EGFR expression. The circle shows the point of the highest Youden index was 0.4. The AUC value amounts to $64 \%$. The determined COP was $44.96 \%$.
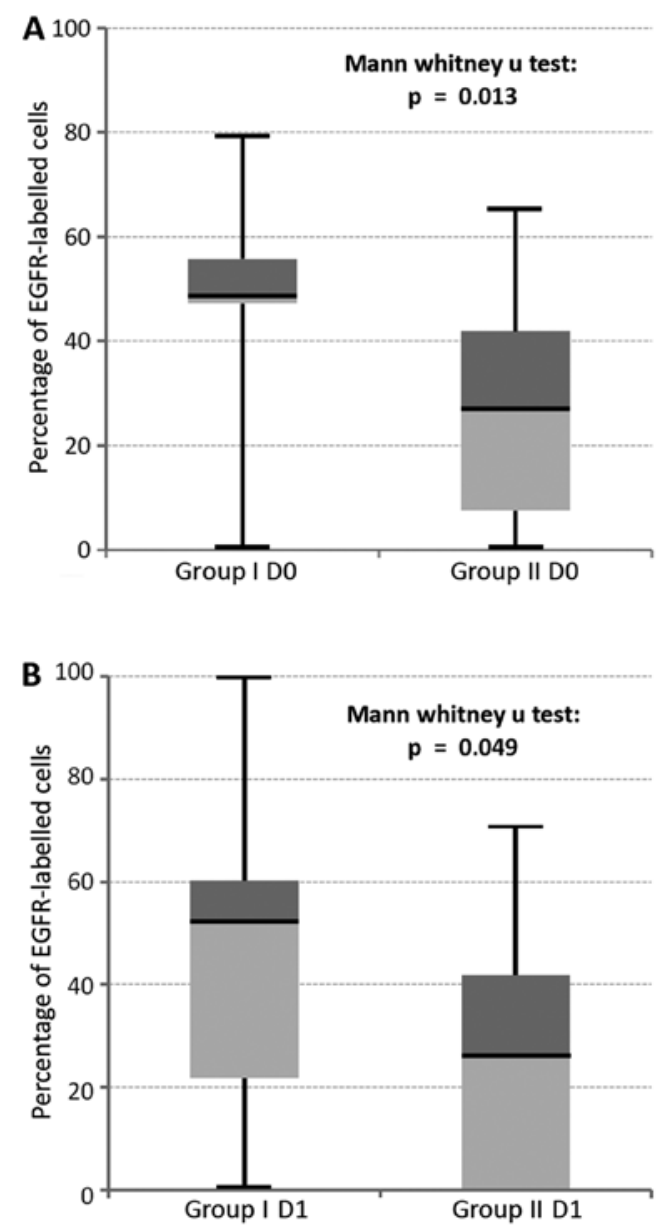

Figure 5. Box-Whisker Plots of EGFR expression in OLP: median values, interquartile range and the standard deviation of labelling indices within the two groups of OLP divided according to their grade of dysplasia are blotted (A) Comparison of lesions of proceeding and non-proceeding lesions without dysplasia. (B) Comparison of lesions of proceeding and non-proceeding lesions with mild dysplasia. The expression was increased in transforming compared to non-transforming OLP in non-dysplastic $(\mathrm{P}=0.013)$ and milddysplastic tissues $(\mathrm{P}=0.049)$. different expression could be proven for low dysplasia lesions. Statistic evaluation revealed that for this lesion the expression was significantly increased in group I compared to group II (D0, P=0.013; D1, P=0.049, Fig. 5).

To display the discriminatory accuracy of the marker for distinguishing between group I and group II, the ROC (receiver operating characteristic) curve was used. It is a plot of the sensitivity (true positive rate) vs. 1-specifity (false positive rate) over all possible threshold values of the marker (Fig. 6). For selection of the optimal threshold value (cut of point) for the biological marker the Youdan index (Y) which is a frequently used summary measure of the ROC curve was calculated. Both measure the effectiveness of a diagnostic marker and enables the selection of an optimal threshold value (cut-off point) for the marker which is associated with the highest Youdan index (Y = Sensitivity + Specificity - 1) (29). The highest Youden index was 0.4. The AUC value amounts to $64 \%$. The optimal threshold value (COP) for distinguishing the transformed from non-transformed lesions was 44.96 (critical expression rate of EGFR).

Using the determined COP the groups were divided into positive and negative lesions where the parameter allows the prediction of the development of a malignancy for individual patients. Out of 53 proceeding OLP 29 (54.7\%) exhibited a labelling index over 44.96. whilst in 'benign' lesions 9 (20\%) showed an expression level above this. The correlation between high risk lesions and the detection of increased expression rates were significant $(\mathrm{P}=0.001$; Table IV).

In addition, the odds ratio and the $95 \%$ confidence interval (CI) could be determined on the results of the cross table. The value of odd ratio was 4.83 . Thus, the risk of cancer development of patients suffering from an OLP exhibiting an expression over the COP of $44.96 \%$ is nearly 5 times higher. The 95\% CI ranges from 1.95 to 11.99 . Thus, the risk of malignant transformation of OLP, which exceeds the threshold of 44.96 is at least double and maximally 12 times higher compared to those which are below the threshold value. 
Table IV. Correlation between overexpression of EGFR (COP=44.96) and malignant transformation of OLP.

\begin{tabular}{lccccc}
\hline & No. of cases & + & - & Positive $(\%)$ & P-value \\
\hline Proceeding OLP & 53 & 29 & 24 & 54.7 & $0.001^{\text {a }}$ \\
Non-proceeding OLP & 45 & 9 & 36 & 20 & \\
\hline
\end{tabular}

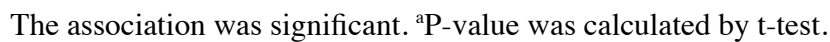

\section{Discussion}

To date, the risk of malignant transformation of OLP lesions are evaluated histopathologically based on the degree of dysplasia (4-6,8-10). This evaluation relies on single biopsies that sometimes are not representative for the whole lesion. Moreover, a high inter-examiner and intra-examiner variability has been identified in the assessment of the degree of epithelial dysplasia $(9,10,30-32)$. It has been stated that objective molecular biological tools are urgently needed to improve the evaluation of the risk of malignant transformation of OLP $(6,7,33)$. Today many markers are discussed, but none of them is either used in clinical routine or can predict the behaviour of OLP on its own $(6,11,13-17,24,27,34-36)$. Therefore, in order to increase accuracy identification of additional reproducible molecular markers by multi-centric studies with larger cohorts for multivariate analyses is urgently needed.

Additionally, only a few studies include a follow up of patients over 5 years $(14,15)$. Hence, there is a lack of information on the rate of malignant transformation of OLP over longer follow-up intervals comparing immunohistochemical features and conventional histopathological aspects of the lesions. Therefore, the present study included only OLP lesions of patients with 5-year follow-up data. Its aim was to evaluate if there were correlations between EGFR overexpression in OLP, the rate of malignant transformation and the histopathological grade of dysplasia.

Previous studies have demonstrated increased EGFR expression in oral leukoplakia $(18,23,24,26,27,37)$. Especially, it has been shown that the percentage of EGFR positive OLP lesions is significantly increased in high-risk sites of the oral cavity, where OSCC often occur $(24,26,27)$. To the best of our knowledge, the present study is the largest one in the field as far as EGFR overexpression is concerned. We were able to include approximately 100 OLP lesions. The main finding is that a relevant overexpression of EGFR was present exclusively in OLP lesions that transformed into OSCC. The correlation between malignant transformation of OLP and EGFR overexpression was statistically significant. Consequently, it seems that in the future prospective studies have to show if routine immunohistochemical determination of EGFR overexpression in OLP allows identifying those lesion that will transform into OSCC. The present study confirms previous findings that concern the relevance of determination of the histopathological grade of dyslasia of OLP for the prediction of malignant transformation. It seems that the histopathological determination of the degree of dysplasia of OLP may have only a limited value for predicting malignant transformation $(18,38)$.
In conclusion, the significant overexpression of EGFR in OLP that transform into OSCC gives information as to the use of the determination of EGFR overexpression in predicting malignant transformation of these lesions. On the other hand, the histopathological determination of the grade of dysplasia of OLP seems to have only a limited value as far as the prediction of malignant transformation is concerned.

\section{Acknowledgements}

The present study has been supported by the DFG (Deutsche Forschungsgemeinschaft, NK 453/3-1). The authors would also like to thank Ms. E. Diebel, Ms. A. Krautheim-Zenk and Ms. S. Schönherr for their valuable technical support.

\section{References}

1. Gondos A, Arndt V, Holleczek B, Stegmaier C, Ziegler H and Brenner H: Cancer survival in Germany and the United States at the beginning of the 21st century: an up-to-date comparison by period analysis. Int J Cancer 121: 395-400, 2007.

2. Jemal A, Siegel R, Xu J and Ward E: Cancer statistics, 2010. CA Cancer J Clin 60: 277-300, 2010

3. Lung T, Tascau OC, Almasan HA and Muresan O: Head and neck cancer, treatment, evolution and post therapeutic survival. Part 2: a decade's results 1993-2002. J Craniomaxillofac Surg 35: 126-131, 2007.

4. Warnakulasuriya S, Johnson NW and van der Waal I: Nomenclature and classification of potentially malignant disorders of the oral mucosa. J Oral Pathol Med 36: 575-580, 2007.

5. van der Waal I: Potentially malignant disorders of the oral and oropharyngeal mucosa; terminology, classification and present concepts of management. Oral Oncol 45: 317-323, 2009.

6. Reibel J: Prognosis of oral pre-malignant lesions: significance of clinical, histopathological, and molecular biological characteristics. Crit Rev Oral Biol Med 14: 47-62, 2003.

7. Pitiyage G, Tilakaratne WM, Tavassoli M and Warnakulasuriya S: Molecular markers in oral epithelial dysplasia: review. J Oral Pathol Med 38: 737-752, 2009.

8. Pindborg JJ, Reichart P, Smith CJ and Waal I: World Health Organization: histological typing of cancer and precancer of the oral mucosa. Springer-Verlag, Berlin, pp47-62, 1997.

9. Warnakulasuriya S, Reibel J, Bouquot J and Dabelsteen E: Oral epithelial dysplasia classification systems: predictive value, utility, weaknesses and scope for improvement. J Oral Pathol Med 37: 127-133, 2008.

10. Fleskens S and Slootweg P: Grading systems in head and neck dysplasia: their prognostic value, weaknesses and utility. Head Neck Oncol 1: 11, 2009.

11. Warnakulasuriya S: Lack of molecular markers to predict malignant potential of oral precancer. J Pathol 190: 407-409, 2000.

12. Ha PK, Chang SS, Glazer CA, Califano JA and Sidransky D: Molecular techniques and genetic alterations in head and neck cancer. Oral Oncol 45: 335-339, 2009.

13. Nasser W, Flechtenmacher C, Holzinger D, Hofele C and Bosch FX: Aberrant expression of p53, p16 ${ }^{\mathrm{INK} 4 \mathrm{a}}$ and Ki-67 as basic biomarker for malignant progression of oral leukoplakias. J Oral Pathol Med 40: 629-635, 2011. 
14. Ries J, Agaimy A, Vairaktaris E, Gorecki P, Neukam FW, Strassburg LH and Nkenke E: Detection of MAGE-A expression predicts malignant transformation of oral leukoplakia. Cancer Invest 30: 495-502, 2012.

15. Ries J, Agaimy A, Vairaktaris E, Kwon Y, Strassburg LH, Neukam FW and Nkenke E: Evaluation of MAGE-A expression and grade of dysplasia for predicting malignant progression of oral leukoplakia. Int J Oncol 41: 1085-1093, 2012.

16. Ries J, Ponader S, Mollaoglu N, Vairaktaris E, Neukam FW and Nkenke E: Early findings of a novel established molecular diagnostic technique for the prediction of malignant transformation in leukoplakia. Mol Med Report 2: 947-952, 2009.

17. Schaaij-Visser TB, Bremmer JF, Braakhuis BJ, Heck AJ, Slijper M, van der Waal I and Brakenhoff RH: Evaluation of cornulin, keratin 4, keratin 13 expression and grade of dysplasia for predicting malignant progression of oral leukoplakia. Oral Oncol 46: 123-127, 2010.

18. Shin DM, Ro JY, Hong WK and Hittelman WN: Dysregulation of epidermal growth factor receptor expression in premalignant lesions during head and neck tumorigenesis. Cancer Res 54 3153-3159, 1994.

19. Smith J, Rattay T, McConkey C, Helliwell $\mathrm{T}$ and Mehanna $\mathrm{H}$ : Biomarkers in dysplasia of the oral cavity: a systematic review. Oral Oncol 45: 647-653, 2009.

20. Vairaktaris E, Serefoglou Z, Avgoustidis D, Yapijakis C, Critselis E, Vylliotis A, Spyridonidou S, et al: Gene polymorphisms related to angiogenesis, inflammation and thrombosis that influence risk for oral cancer. Oral Oncol 45: 247-253, 2009.

21. Vairaktaris E, Yapijakis C, Derka S, Vassiliou S, Serefoglou Z, Vylliotis A, Wiltfang J, et al: Association of platelet glycoprotein Ia polymorphism with minor increase of risk for oral cancer. Eur J Surg Oncol 32: 455-457, 2006.

22. Yapijakis C, Vairaktaris E, Vassiliou S, Vylliotis A, Nkenke E, Nixon AM, Derka S, et al: The low VEGF production allele of the $+936 \mathrm{C} / \mathrm{T}$ polymorphism is strongly associated with increased risk for oral cancer. J Cancer Res Clin Oncol 133: 787-791, 2007.

23. Oliveira LR and Ribeiro-Silva A: Prognostic significance of immunohistochemical biomarkers in oral squamous cell carcinoma. Int J Oral Maxillofac Surg 40: 298-307, 2011.

24. Ribeiro DC, Gleber-Netto FO, Sousa SF, Bernardes VD, Guimaraes-Abreu MH and Aguiar MC: Immunohistochemical expression of EGFR in oral leukoplakia: association with clinicopathological features and cellular proliferation. Med Oral Patol Oral Cir Bucal 17: e739-744, 2012.

25. Nagatsuka H, Ishiwari Y, Tsujigiwa $H$, Nakano $K$ and Nagai N: Quantitation of epidermal growth factor receptor gene amplification by competitive polymerase chain reaction in pre-malignant and malignant oral epithelial lesions. Oral Oncol 37: 599-604, 2001.
26. Taoudi Benchekroun M, Saintigny P, Thomas SM, et al: Epidermal growth factor receptor expression and gene copy number in the risk of oral cancer. Cancer Prev Res 3: 800-809, 2010.

27. Srinivasan M and Jewell SD: Evaluation of TGF-alpha and EGFR expression in oral leukoplakia and oral submucous fibrosis by quantitative immunohistochemistry. Oncology 61: 284-292, 2001.

28. Srinivasan M and Jewell SD: Quantitative estimation of PCNA, $c-m y c$, EGFR and TGF- $\alpha$ in oral submucous fibrosis - an immunohistochemical study. Oral Oncol 37: 461-467, 2001.

29. Fluss R, Faraggi D and Reiser B: Estimation of the Youden Index and its associated cutoff point. Biom J 47: 458-472, 2005.

30. Brennan M, Migliorati CA, Lockhart PB, Wray D, Al-Hashimi I, Axell T, Bruce AJ, et al: Management of oral epithelial dysplasia: a review. Oral Surg Oral Med Oral Pathol Oral Radiol Endod 103 (Suppl 19): S19.e1-S19.e12,2007.

31. Fleskens SA, Bergshoeff VE, Voogd AC, van Velthuysen ML, Bot FJ, Speel EJ, Kremer B, et al: Interobserver variability of laryngeal mucosal premalignant lesions: a histopathological evaluation. Mod Pathol 24: 892-898, 2011.

32. Holmstrup P, Vedtofte P, Reibel J and Stoltze K: Oral premalignant lesions: is a biopsy reliable? J Oral Pathol Med 36: 262-266, 2007.

33. Napier SS and Speight PM: Natural history of potentially malignant oral lesions and conditions: an overview of the literature. J Oral Pathol Med 37: 1-10, 2008.

34. Angiero F, Berenzi A, Benetti A, Rossi E, Del Sordo R, Sidoni A, Stefani M and Dessy E: Expression of p16, p53 and Ki-67 proteins in the progression of epithelial dysplasia of the oral cavity. Anticancer Res 28: 2535-2539, 2008.

35. Cruz IB, Snijders PJ, Meijer CJ, Braakhuis BJ, Snow GB, Walboomers JM and van der Waal I: p53 expression above the basal cell layer in oral mucosa is an early event of malignant transformation and has predictive value for developing oral squamous cell carcinoma. J Pathol 184: 360-368, 1998.

36. Feng CJ, Li HJ, Li JN, Lu YJ and Liao GQ: Expression of Mcm7 and Cdc6 in oral squamous cell carcinoma and precancerous lesions. Anticancer Res 28: 3763-3769, 2008.

37. Grandis JR and Tweardy DJ: TGF-alpha and EGFR in head and neck cancer. J Cell Biochem (Suppl 17): 188-191, 1993.

38. Rautava J, Jee KJ, Miettinen PJ, Nagy B, Myllykangas S, Odell EW, Soukka T, et al: ERBB receptors in developing, dysplastic and malignant oral epithelia. Oral Oncol 44: 227-235, 2008. 